\title{
ANALYSIS OF UT1 DETERMINATIONS BY VLBI WITHIN PROJECT IRIS
}

\author{
H. Schuh \\ Geodetic Institute \\ University of Bonn \\ Nussallee 17 \\ D-5300 Bonn 1, FRG
}

\begin{abstract}
Two series of UT1 data determined by VLBI within Project IRIS at 5 day intervals respectively on a daily basis have been subjected to an iterative spectral analysis. Various significant oscillations with periods less than two months could be detected in the IRIS UT1 data. Most of the periods between 18 and 63 days obviously correspond to short-period variations in global atmospheric data. The other significant variations with periods shorter than 35 days can be clearly identified as being due to the influence of zonal tides on the rotation of the earth. The results of the spectral analysis are compared with the theoretical periods and phases and with the amplitudes given by Yoder et al. (1981). The spectral analysis reveals very clearly the monthly (27.6d) and the fortnightly (13.7d) terms and also the smaller variations at 14.77 days and 9.13 days. A similar analysis of 8 subsets, each with two months of the daily UT1 determinations, indicates that the small 7.10 day term can already be extracted from the VLBI observations.
\end{abstract}

\section{INTRODUCTION}

Atmospheric excitation causes several periodic fluctuations in the earth's rotation which can be expressed either in changes of UT1 (Universal Time 1) or of LOD (Length of Day). While the variations in the mean frequency range like the annual and the semiannual oscillation are relatively large and therefore easy to detect in UT1 observations, the inferred short-period terms ( $\leq 2$ months) are much smaller and are very close to the detection limit, even for today's high-precision observation methods. Additionally, there are several short-period variations in UT1 between 5 and 35 days - which are due to the influence of zonal tides on the rotation of the earth. Although theoretically well-known, most of these small oscillations are below the accuracy of the majority of the UT1 observation methods. Ten of these variations are larger than +-0.04 msecs, four amplitudes are larger than 0.1 msecs. Only the amplitudes of the main fortnightly variation (13.66 days) and the main monthly variation ( 27.56 days) are close to $1 \mathrm{msec}$ and only these variations could be extracted from astronomical UT1 observations. 
Currently, the modern space techniques are the main tools for the ERP (Earth Rotation Parameters) determination. In this paper an analysis of the UT1 determinations by VLBI within the project IRIS will be presented.

\section{DETERMINATION OF THE EARTH ROTATION PARAMERTERS WITHIN PROJECT IRIS}

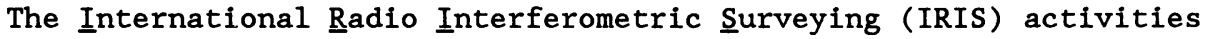
consist of VLBI observing sessions designed to monitor UT1 and polar motion. The experiments are 24 hours in duration at five-day intervals. The IRIS observations normally involve three stations in the United States (the Westford radiotelescope in Massachusetts, the George $R$. Agassiz station in Texas and the Richmond Observatory in Florida) and the $20 \mathrm{~m}$ radiotelescope of the Fundamental Station Wettzell in the Federal Republic of Germany. One session per month also includes the Onsala Space Observatory in Sweden. Thus, since Jan. 5th, 1984, the date that the Wettzell Observatory began regular operations, the IRIS system has been routinely providing the $\mathrm{x}$ and $\mathrm{y}$ components of polar motion with an accuracy of better than +-1 marcsec, and UT1 to +-0.05 msecs. The UT1 series from IRIS between Jan. 1984 and July 1986, published in the monthly IRIS Bulletin A, contains 175 data points at 5-day intervals covering a period of 2.5 years. It will be called 'data set $1^{\prime}$ ' in the following chapters. Of course, the long-term trend has to be removed, in order to make the periodic variations in UT1 visible. These can be seen on figure 1 , showing the residuals after subtracting a best-fit second order polynomial.

Since April 1985 short VLBI experiments on the baseline Westford to Wettzell have been performed in order to monitor UT1 every day with an accuracy of better than $0.1 \mathrm{msec}$. By the combination of these daily results between 1st of April, 1985 and 23th of July, 1986 with the high-precision 5-day IRIS data, a consistent data set was produced covering a time period of one year and four months. A straight line was fit to remove the linear trend in this second data set ('data set $2^{\prime}$ ).

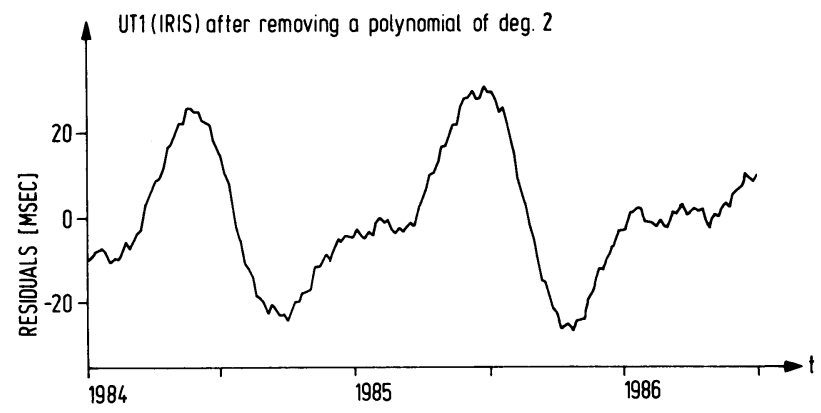

Figure 1. Residuals of a second order polynomial fit of the IRIS UT1 determinations (data set 1 ), showing the annual and semiannual oscillations and additional short-period variations 
For both data sets interpolation methods were used in order to replace a few missing data points and - in the case of data set 2 - to produce equidistant data. Then, the two data sets have been subjected to an iterative spectral analysis of which results will be presented in the next chapters.

\section{SHORT PERIODS IN THE IRIS UT1 DETERMINATIONS}

\subsection{Results of the iterative spectral analysis}

In each iteration of this analysis the power spectrum is computed and the frequency of the maximum peak is extracted. Then a least-squares fit is carried out, entering all periods which have been determined until the current iteration and solving for their amplitudes and phases. The residuals are used to compute the power spectrum of the next iteration. of course, the residuals cannot be rigorously considered as uncorrelated observations, but with this data type the effect is negligible (Schuh, 1981). In that reference the method, which has been proved to be very effective for a spectral decomposition of the BIH UT1 data, has been described in detail.

The spectral analysis revealed the well-known annual and the semiannual oscillations as well as the periods around four months and around three months. Additionally several variations in the period range from 63 to 18 days, which correspond to short-period variations in global atmospheric data, were found by the spectral decomposition of the IRIS UT1 determinations. Variations with periods of 40 to 60 days in atmospheric data had been reported first by Madden and Julian (1971, 1972). Lambeck and Cazenave (1974) presumed that similar to the excitation of the earth's rotation by the global annual and semiannual atmospheric behaviour, there should exist an atmospheric forcing on the rotation of the earth with a $\sim 50$ day period. Rosen and Salstein (1983) confirmed the existence of fluctuations with periods of $\sim 50$ days from a spectral analysis of the time series of the global atmosphere's angular momentum, constructed from several years of zonal wind data. Their power spectra additionally showed significant shorter periods, in particular near 30 days and 17 days. Recent investigations by Hara and Yokoyama (1985) revealed high coherence between atmospheric angular momentum data and UT1 observations for periods of about 50 days, 32-36 days, 24 days and 16-21 days.

In table I the results from our spectral analysis of the IRIS-data are compared with the periods in the atmosphere's angular momentum, reported by Rosen and Salstein (1983) and by Hara and Yokoyama (1985). It can be seen that all the UT1 oscillations related to the atmosphere change in both period and amplitude, which shows the unsteady and transient feature of these periodicities. As usual for this kind of frequency analysis, time variant fluctuations are expressed by several periods, for example 42, 49, 55, 63 days to describe the irregular '40-60 day' fluctuation. 
TABLE I: Results of spectral analysis of the 5 day IRIS UT1 determinations (data set 1 ) and the 1 day IRIS+Intensive UT1 determinations (data set 2) for periods less than 63 days compared with periods detected in global atmospheric data

\begin{tabular}{|c|c|c|c|}
\hline \multicolumn{2}{|c|}{ observed variations of UT 1} & \multicolumn{2}{|c|}{$\begin{array}{l}\text { variations of global atmospheric } \\
\text { data }\end{array}$} \\
\hline $\begin{array}{l}\text { 5d IRIS } \\
P \text { [d] }\end{array}$ & $\begin{array}{l}\text { Id IRIS+ Intensive } \\
\text { P[d] }\end{array}$ & $\begin{array}{c}\text { ROSEN and } \\
\text { SALSTEIN (1983) }\end{array}$ & $\begin{array}{l}\text { HARA and } \\
\text { YOKOYAMA (1985) }\end{array}$ \\
\hline 62 & 63 & & \\
\hline 57 & 55 & & \\
\hline 53 & & & \\
\hline 51 & 49 & -50 & -50 \\
\hline & 42 & & \\
\hline 34 & 35 & -30 & $32-36$ \\
\hline & 25 & & 24 \\
\hline & 21 & $\sim 17$ & $16-21$ \\
\hline
\end{tabular}

All other variations with periods shorter than 35 days are due to the influence of earth tides on the rotation of the earth. These variations caused by the tidal deformation of the polar moment of inertia have been theoretically derived by several authors, for example by Woolard (1959) and by Yoder et al. (1981). The periods and phases of these components of the earth's rotation are well determined by the motions of the moon and the sun, but the amplitudes are uncertain because of the imperfections in the model for the tidal deformations of the liquid core and the oceans and because of the probable inelasticity of the earth's mantle (Zschau and Wang, 1986), which has not been taken into account in previous investigations.

On the right hand side of tables IIa,b the results of the spectral analysis are compared with the theoretical periods and phases and with the amplitudes given by Yoder et al. (1981). The analysis of the 5 day IRIS UT1 determinations reveals the main fortnightly term ( 13.7 days) and the main monthly period (27.6 days) very clearly (see table IIa). The agreement between the results of our analysis and theory is not so good for the 31.8 day period - probably because of some correlations with the atmospheric variations in the same period range.

It should be noted here that from the combined data set 2 of the 5 day IRIS and the ('intensive') daily UT1 determinations (table IIb), additionally the periods of 14.77 days and of 9.13 days could be extracted very exactly. But again, there are some difficulties to resolve the 31.8 day period: only a small variation at 30.4 days was found.

The power spectrum of data set 2 is shown on figure $2 a$. There is no significant peak to be seen except for the dominating annual and for the semiannual period. But after five iterations (figure $2 b$ ), the power spectrum reveals two sharp peaks at 13.7 days and at 27.6 days as well as a broader peak representing the 40-60 day fluctuation. Another eight iterations later (figure $2 \mathrm{c}$ ) the peaks at 9.13 days and at 14.77 days appear and also two broader peaks around 25 days and around 35 days. Please note the different scales of the three power spectra. 
TABLES IIa,b. Results of least-squares fit of the 5 day IRIS UT1 determinations and of the daily IRIS+Intensive UT1 determinations for periods less than 35 days (periods derived from spectral analysis), compared with the theoretical tidal-induced UT1 variations. The errors of the phases and of the amplitudes are twice the formal errors.

Tidal-induced UT1-variations observed by VLBI within project IRIS Results of spectrol analysis

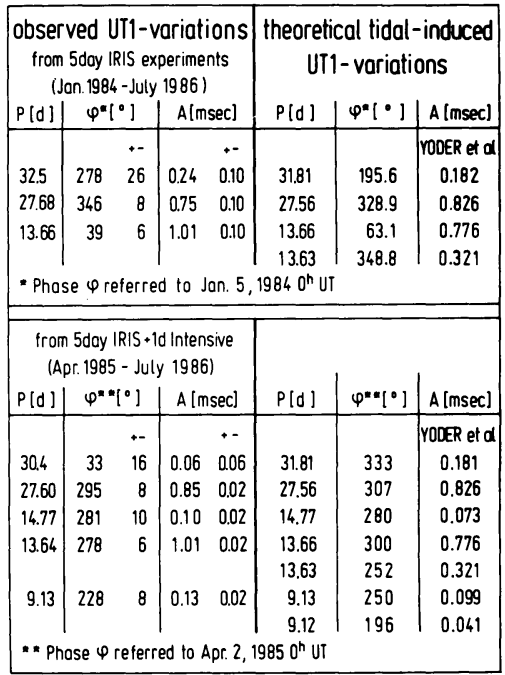

TABLE IIa

POWER SPECTRA FROM ANALYSIS OF DAILY UT1DETERMINATIONS (APR. 1985-JULY 1986)

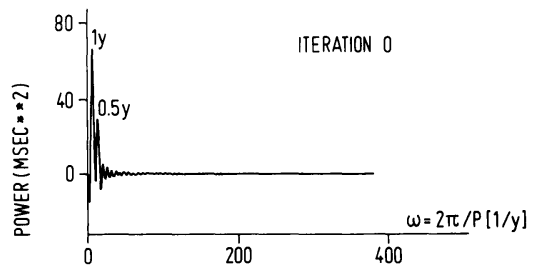

Figure 2a. Power spectrum of the original data set 2

Figure 2b. Power spectrum of iteration 5 of the analysis
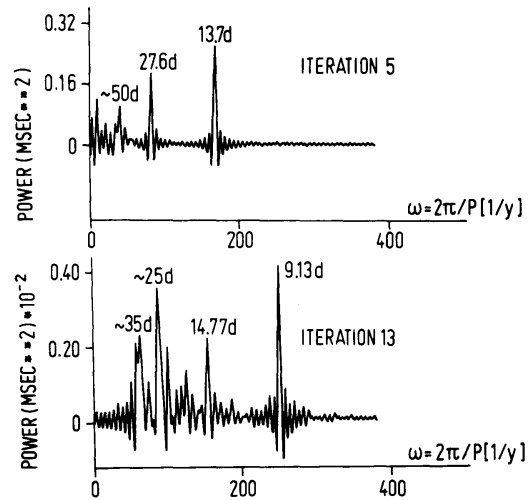

Figure 2c. Power spectrum of iteration 13 of the analysis 


\subsection{Analysis of subsets of the daily UT1 determinations}

The spectral analysis of data set 2 revealed several significant frequencies in the mean period range and in the short period range of up to 9.13 days. However, to investigate the very high frequencies, i.e. periods less than 14 days, shorter data sets are more appropriate, because systematic trends and long periods can be filtered out more easily without biasing the very short periods.

Therefore, the whole data set 2 was splitted up into 8 subsets, each covering a time period of 2 months. The iterative spectral analysis was repeated for each of the subsets after having removed the linear trend by a best-fit straight line. In each analysis, the monthly and the fortnightly variations were found as well as one period between 46 and 64 days, which can be related to the variable '40-60 day' period in the atmosphere's angular momentum. Those significant periods, which could be detected additionally in each of the subsets, are plotted on figure 3 . The tidal-induced 9.13 day period is confirmed from this analysis as well as the existence of a variation around 21 days probably due to atmospheric excitation. Moreover, there seems to be some signal around 7.1 days. According to Yoder et al. (1981), the highly correlated periods of 7.09 and 7.10 days, which are the largest terms below the 9.13 day oscillation, have a combined amplitude of only $0.017 \mathrm{msecs}$. In spite of this marginally detectable amplitude, the 7.1 day period seems already to appear in the IRIS UT1 determinations. Two power spectra of the analysis of subset 8 (June 1986 - July 1986) of data set 2 are plotted on figures $4 \mathrm{a}, \mathrm{b}$. In iteration 4 the peak at 9.13 days is clearly visible, whereas in iteration 9 there is obviously some power around 7.1 days.

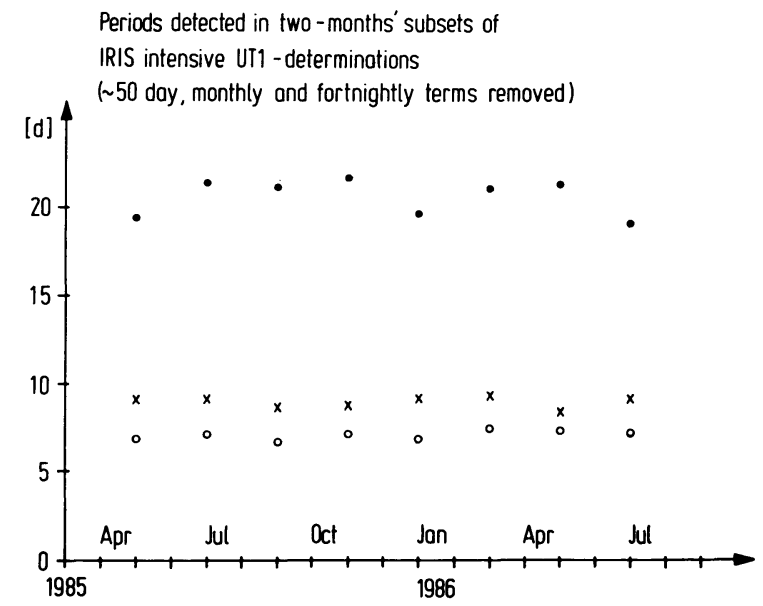

Figure 3. Periods detected from spectral analysis of subsets of the IRIS+Intensive UT1 data, each covering 2 months 
POWER SPECTRA FROM ANALYSIS OF DALLY UT1-

DETERMINATIONS(JUNE 1986 -JULY 1986)
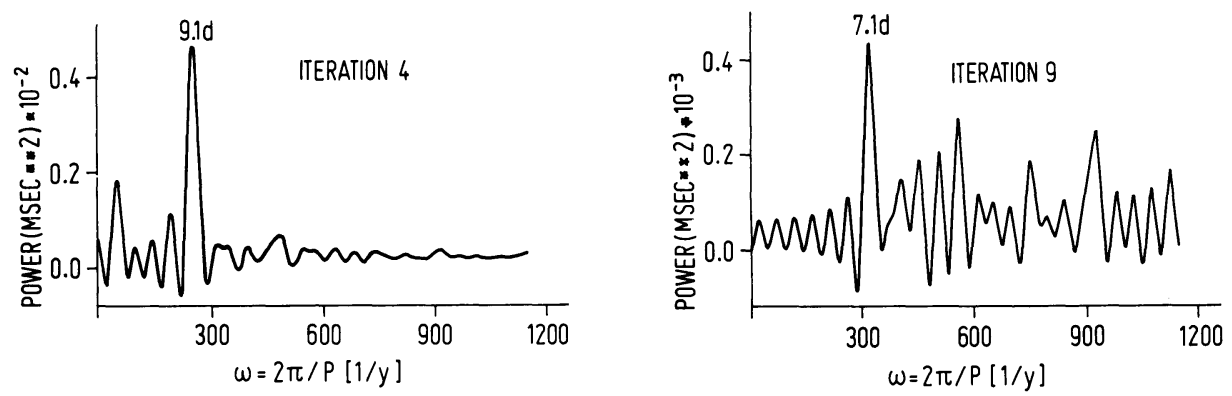

Figure $4 \mathrm{a}, \mathrm{b}$. Power spectra at two iterations of subset 8 (June/July, 86)

\subsection{Analysis using the theoretical periods}

It has to be emphasized, that no a priori knowledge about the frequencies detected has been used in this method of frequency analysis. Therefore the presented results constitute a statistical and purely empirical confirmation of the short-period oscillations in UT1 which are caused by the earth tides and by atmospheric excitation of the earth's rotation.

Of course, neither data set 1 nor data set 2 is long enough to resolve the 13.63 day period which is very close to the 13.66 days, because the only frequencies which are independent are those which are spaced by the Fourier frequency. In the case of the daily UT1 determinations this would mean that a data set about 10 times longer than the existing one is needed to separate those two close oscillations. On table IIb it can be seen that the only fortnightly period (which was determined to 13.64 days) absorbed the total power in this range.

In order to estimate how the power is distributed between the two periods (13.63d and 13.66d) a further solution was carried out where the theoretical periods of the six largest tidal-induced variations ( 9.13 , $13.63,13.66,14.77,27.56,31.81$ ) and the 7.10 day period have been entered in the least-squares fit of data set 2 solving again for phases and amplitudes. All other periodic fluctuations in the data are determined as usual by spectral analysis and removed from the data. The results for the periods concerned, are shown on table III. We can observe that the total power in the fortnightly range has in fact been distributed to the 13.66 and the 13.63 day terms, although these results received very high formal errors due to the strong correlation of the two close periods.

Again, the 14.77 day and the 9.13 day terms agree very well with theory in both phase and amplitude. But it is hard to decide, if the 7.10 day period is really contained in the UT1 data because of its small amplitude, although there is a good agreement with the theoretical phase and amplitude given by Yoder et al. (1981). 
TABLE III. Results of least-squares fit of the IRIS+Intensive UT1 determinations (data set 2) for periods less than 35 days (theoretical periods entered), compared with the theoretical tidal-induced UT1 variations.

\begin{tabular}{|c|c|c|c|c|c|c|}
\hline \multirow{2}{*}{$\begin{array}{c}\text { theoretical } \\
\text { periods } \\
\text { entered } \\
P[d] \\
\end{array}$} & \multicolumn{3}{|c|}{$\begin{array}{l}\text { observed values } \\
\text { (IRIS+Intensive) }\end{array}$} & \multicolumn{3}{|c|}{$\begin{array}{l}\text { theoretical tidal-induced } \\
\text { UI1-variations }\end{array}$} \\
\hline & $\varphi^{n}\left[0^{0}\right]$ & $A[\mathrm{~ms}$ & sec] & $P[d]$ & $\varphi^{*}\left[0^{\circ}\right]$ & A[msec] \\
\hline & +- & & + & & & YODER et al. \\
\hline 31.81 & 16446 & 0.03 & 0.02 & 31.81 & 333 & 0.182 \\
\hline 27.56 & 2912 & 0.87 & 0.02 & 27.56 & 307 & 0.826 \\
\hline 14.77 & 27612 & 0.10 & 0.02 & 14.77 & 280 & 0.073 \\
\hline 13.66 & 2808 & 0.96 & 0.14 & 13.66 & 300 & 0.776 \\
\hline 13.63 & 32382 & 0.10 & 0.14 & 13.63 & 252 & 0.321 \\
\hline \multirow[t]{2}{*}{9.13} & 22810 & 0.13 & 0.02 & 9.13 & 250 & 0.099 \\
\hline & & & & 9.12 & 196 & 0.041 \\
\hline \multirow[t]{2}{*}{7.10} & 21480 & 0.014 & 0.02 & 7.10 & 221 & 0.012 \\
\hline & & & & 7.09 & 171 & 0.005 \\
\hline
\end{tabular}

- Phose $\varphi$ referred to Apr. 2nd, 1985, 0h UT

\section{CONCLUSIONS AND FUTURE ASPECTS}

An analysis of the 5-day UT1 values obtained within the IRIS campaign since Jan. 1984 and of the daily UT1 results obtained within the IRIS intensive sessions since April 1985 has produced promising results. In particular the daily intensive sessions between Westford and Wettzell have considerably improved the resolution of the frequencies to be detected and allow to investigate the very small, short-period UT1 variations, caused by the atmosphere and by the earth tides. Furthermore the intensive observations make an investigation of the small variations with periods shorter than 10 days possible, like the 9.13 day and the 7.10 day terms. Thus, by the continuation of the daily UT1 observations and by a further improvement of the VLBI hardware and software it will be possible in the future to separate even the smaller terms of tidal-induced UT1 variations by VLBI and to compare their amplitudes with those observed by other techniques as well as those predicted by theory.

\section{REFERENCES}

Hara, T. and Yokoyama, K.: 'A Synoptic Analysis of Short Period Fluctuations of UT1', Proceedings of the International Conference on Earth Rotation and the Terrestrial Reference Frame, Columbus, Ohio, Vo1. 2, 490-504, 1985.

Lambeck, K. and Cazenave, A.: 'The earth's rotation and atmospheric circulation' II, The continuum, Geophys. Journ. Roy. Astron. Soc., No. 38, 49-61, 1974.

Madden, R.A. and Julian, P.R.: 'Detection of a 40-50 day oscillation in the zonal wind in the tropical Pacific', J. Atmos. Sci., 28, $702-708,1971$.

Rosen, R.D. and Salstein, D.A.: 'Variations in Atmospheric Angular Mo- 
mentum on Global and Regional Scales and the Length of Day', $\mathrm{J}$. of Geophys. Res., 88, No.C9, 5451-5470, 1983.

Schuh, H.: 'Zur Spektralanalyse von Erdrotationsschwankungen', Veroeff. d. Bayer. Komm. f. d. Intern. Erdm. d. Bayer. Ak. d. Wiss., No. 41, 176-193, Munich, 1981.

Woolard E.W.: 'Inequalities in mean solar time from tidal variations in the rotation of the earth', Astron. Journ., 64, No. 1269, 140-142, 1959.

Yoder, C.F., Williams, J.G. and Parke, M.E.: 'Tidal Variations of Earth Rotation', J. of Geophys. Res., 86, 881-891, 1981.

Zschau, J. and Wang, R.: 'Incomplete Elasticity in the Earth's Mantle, Consequences for Earth Tides', Joint Meeting of the European Geophys. Soc. and the Europ. Seismo1. Comm., Aug. 1986, Kiel, FGR, abstract in Terra cognita, Vo1. 6, No. 3, 464, Summer 1986.

ACKNOWLEDGEMENTS. The author wishes to express his gratitude to all the many organizations and individuals that have contributed to the IRIS project. This work has been performed as part of activities of the Special Research Group on Satellite Geodesy (SFB 78) sponsored by the DFG (Deutsche Forschungsgemeinschaft).

\section{DISCUSSION}

F.W. Fallon: In fitting $5 \mathrm{~d}$ and $1 \mathrm{~d}$ series together and filling missing points, what kind of interpolation did you use?

H. Schuh: A linear interpolation was totally sufficient, because most of the data points had only to be shifted by a few hours.

F.W. Fallon: Did you remove all Yoder tidal terms first?

H. Schuh: No.

C.F. Yoder: Have you considered the use of integrated AAM to remove the atmospheric signal?

H. Schuh: Of course, it is possible to remove either the fluctuations due to atmospheric excitation or the theoretical tidal-induced variations in advance. By this separation you can reduce possible correlations of the two effects, for example in the period range of 30-32 days. However by modelling these contributions you also introduce an additional error or at least additional noise which can affect the results. And there are several other effects which cause periodic variations in the earth's rotation like the ocean tides or groundwater fluctuations which would have to be modelled, too. Therefore, the principal idea was to analyze the high-precision VLBI data first, without any a priori treatment of the UT1 data.

$R$. Eanes: In deriving conclusions about the scaling factor $\mathrm{k} / \mathrm{C}$ for monthly and fortnightly tides care must be used to separate terms with frequencies that are different only by one cycle in 18.6 years.

H. Schuh: I completely agree. For the derivation of frequency dependent Love numbers it is necessary to look at all periods in the fortnightly and in the monthly range and not only at the two main terms as has been done in several publications. 


\section{DISCUSSION}

Dickey: We have noticed systematic differences between the IRIS daily and 5-day values, a 0.1 msec offset plus possible additional periodic signatures. Have you considered these? Would they affect your analysis?

Reply by Schuh: We have removed the small offset between the two data sets before starting the analysis. The additional signature which can be seen in the daily UT1 determinations with respect to the IRIS 5-day values shows the quality of the daily data and the necessity for daily UT1 observations. Due to the higher resolution, the daily UT1 series contains more information in the short period range than the 5-day data. For example, it allowed us to extract the 14.77 and the 9.13 day periods which could not be seen in the 5-day UT1 series. 